\title{
Squamous cell carcinoma antigen 1 and 2 expression in cultured normal peripheral blood mononuclear cells and in vulvar squamous cell carcinoma
}

\author{
Magdalena Chechlinska • Magdalena Kowalewska • Edyta Brzoska-Wojtowicz • \\ Jakub Radziszewski • Konrad Ptaszynski • Janusz Rys • Janina Kaminska • \\ Radoslawa Nowak
}

Received: 13 April 2010 / Accepted: 10 June 2010 /Published online: 1 July 2010

(C) The Author(s) 2010. This article is published with open access at Springerlink.com

\begin{abstract}
Squamous cell carcinoma antigen (SCCA) is expressed in normal squamous cell epithelia and in squamous cell carcinomas (SCC). Two nearly identical genes encode the inhibitory serpins SCCA1 (SERPINB3) and SCCA2 (SERPINB4). Serum levels of SCCA are elevated in patients with benign skin diseases and in patients with SCC. SCCA, used for the monitoring of SCC patients, presents no satisfactory diagnostic specificity. As we have shown previously, the reverse transcription polymerase chain reaction (RT-PCR)-based SCCA messenger RNA (mRNA) testing aimed at detecting disseminated cancer cells may be hampered by the false-positive results due to SCCA expression in activated peripheral blood mononuclear cells (PBMC). The aim of this study was to
\end{abstract}

\footnotetext{
M. Chechlinska $(\bowtie)$

Department of Immunology, Maria Sklodowska-Curie Memorial Cancer Centre and Institute of Oncology,

Roentgena 5,

02-781 Warsaw, Poland

e-mail: chech@coi.waw.pl

M. Kowalewska $\cdot$ R. Nowak

Department of Molecular Biology, Maria Sklodowska-Curie

Memorial Cancer Centre and Institute of Oncology,

Roentgena 5,

02-781 Warsaw, Poland

E. Brzoska-Wojtowicz

Department of Cytology, Institute of Zoology, Biology Faculty,

Warsaw University,

Miecznikowa 1,

02-096 Warsaw, Poland

\section{J. Radziszewski}

Department of Brachytherapy, Maria Sklodowska-Curie Memorial

Cancer Centre and Institute of Oncology,

Roentgena 5,

02-781 Warsaw, Poland
}

assess the expression of SCCA at mRNA and protein levels in cultured normal PBMC, compared to that in vulvar SCC (VSCC) samples. High SCCA concentrations were found in vulvar tumours and in metastatic lymph nodes, while negative inguinal lymph nodes from the same patients often presented significantly less SCCA. In normal activated PBMC, the level of SCCA protein was the lowest. At the mRNA level SCCA was detectable in normal PBMC even in cultures with no mitogen stimulation, but only by the nested RT-PCR, contrary to VSCC samples found to be SCCA positive already in one-step PCR. Both SCCA1 and SCCA2 transcripts were present in cultured PBMC; SCCA1 was expressed at a higher level than SCCA2. In conclusion, both SCCA forms are detectable in normal

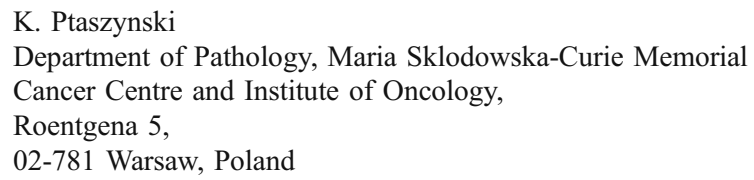

J. Kaminska

Department of Tumour Markers, Maria Sklodowska-Curie

Memorial Cancer Centre and Institute of Oncology,

Roentgena 5,

02-781 Warsaw, Poland 
PBMC cultured in vitro. SCCA expression level in normal PBMC is much lower than in the squamous epitheliumderived cells. In VSCC, in addition to tumour itself, metastatic lymph nodes seem also to be a potential source of serum SCCA.

Keywords SCC · SERPINB3 - SERPINB4 ·

Tumour marker $\cdot$ PBMC $\cdot$ Vulvar carcinoma

\section{Introduction}

Squamous cell carcinoma antigen (SCCA), first discovered in the squamous cell carcinoma of the cervix [1], is expressed in different squamous cell carcinomas (SCC) and in normal squamous cell epithelia. SCCA belongs to the family of inhibitory serine protease inhibitors (serpins), involved in apoptosis, cell migration and invasiveness [2].

Elevated levels of serum SCCA proteins are common in patients with benign, inflammatory skin diseases, such as psoriasis and atopic dermatitis, as well as in patients with squamous cell carcinomas, especially of the cervix, head and neck, lung, oesophagus and vulva, where SCCA concentrations may correlate with clinical stage, histological grade and tumour burden [1,3-7]. Serum SCCA has an independent prognostic value in patients with cervical carcinoma and with head and neck malignancies $[6,8]$ and is used for the diagnosis and monitoring of patients with SCC [9]. There are two SCCA proteins, encoded by two, nearly identical genes, SCCA1 (SERPINB3) and SCCA2 (SERPINB4). SCCA1 and SCCA2 proteins, although they present $92 \%$ amino acid sequence homology and target different proteases. The neutral SCCA1 inhibits papaine-like lysosomal cysteine proteases, cathepsine S, L and $\mathrm{K}$, and the acidic SCCA2 inhibits the serine proteases, cathepsine $\mathrm{G}$ and mast cell chymase $[10,11]$.

Both SCCA proteins are colocalised in normal and malignant squamous epithelial cells, but the dominating serum form in patients with SCC is SCCA2 [12-14]. Elevated SCCA2/SCCA1 messenger RNA (mRNA) ratio in primary SCC of the head and neck and of cervix was found to be an independent predictor of tumour recurrence $[15,16]$. SCCA is also regarded as a molecular tumour marker for disseminated SCC cell detection [17]. However, as we have previously shown while studying the question of false-positive reverse transcription polymerase chain reaction (RT-PCR) results of disseminated cancer cell detection [18], there is an expression of a number of the so-called tumourspecific molecular markers, including SCCA, in activated peripheral blood mononuclear cells (PBMC).

Here, we aimed to study the total SCCA as well as SCCA1 and SCCA2 expression at both the mRNA and protein levels, in normal unstimulated and PHA-stimulated
PBMC, and to compare the obtained total SCCA protein expression to that in vulvar squamous cell carcinoma (VSCC), one of many types of SCCs.

\section{Material and methods}

PBMC and cell lines

PBMC of six healthy donors were obtained by standard Ficoll-Paque (Pharmacia) gradient centrifugation; samples of uncultured cells were taken, and the remaining cells were cultured in a standard medium supplemented with $10 \%$ fetal calf serum (FCS) and gentamycin, for 1 day without mitogen, and in paralell, for $1-6$ days in the presence of PHA $(1 \mu \mathrm{g} / \mathrm{ml})$. Cultures were centrifuged, supernatants were collected and frozen, cells were washed twice in PBS and the cell pellet was kept frozen at $-70^{\circ} \mathrm{C}$ until RNA was isolated.

Squamous epithelial cell lines, the epidermoid vulvar carcinoma cell line, A431 (Deutsche Sammlung von Zellkulturen und Mikroorganismen, Braunschweig, Germany), the human cervical carcinoma cell line, SKGIIIa (RINKEN BRC, National Bio-Resource Project of the MEXT, Japan), the supraglottic larynx carcinoma cell line, UT-SCC-8 (The University of Turku, Finland), and the human keratinocyte cell line, HaCat [19], as well as the cervix adenocarcinoma cell line, HeLa (Warsaw Medical University, Warsaw Poland), were cultured at $37^{\circ} \mathrm{C}, 5 \%$ $\mathrm{CO}_{2}$ in the standard media supplemented with $10 \% \mathrm{FCS}$ and gentamycin.

Post-surgical samples from vulvar carcinoma patients

We studied the samples obtained from surgical specimens of 41 patients treated in the Maria Sklodowska-Curie Memorial Cancer Center and Institute of Oncology for VSCC (pT1-2, N0-2, M0). All patients gave their informed consent. Samples including 39 tumours, 15 regional lymph nodes and 36 uninvolved adjacent tissues were snap-frozen and stored at $-70^{\circ} \mathrm{C}$ until they were pulverised on liquid nitrogen, using a Microdismembrator II (B Braun Biotech International, Melsungen, Germany). The uninvolved tissues comprised cross-sections of skin next to the surgical margin. Histologically, they were composed of dermis and the epidermal layer.

\section{RNA isolation and RT-PCR}

Total RNA was isolated from PBMC at each time point (0, before culture; unstimulated 1-day cultures; and PHAstimulated 1-6-day cultures ) and from pulverised postsurgical samples from VSCC patients with the use of 
Quiagen RNeasy kits. The quantity and quality of the RNA that was obtained were examined by spectrophotometry (Nanodrop, Thermoscientific) and by denaturing gel electrophoresis with ethidium bromide. Two micrograms of the total RNA was reverse transcribed with random hexamer primers, in the final volume of $20 \mu \mathrm{l}$, using SuperScript reverse transcriptase (Invitrogen), according to the manufacturer's instructions. The quality of complementary DNA preparations was controlled by the PCR of DNA polymerase $\beta$, as described previously [18]. Total SCCA, SCCA1 and SSCA2 expression was analysed in PBMC by the nested RT-PCR with specific primers. Tissue samples obtained from VSCC patients were examined for total SCCA expression by one-step RT-PCR.

Measurements of SCCA protein expression

Total cell protein was extracted from unstimulated and stimulated PBMC of four normal donors and from pulverised post-surgical samples of seven patients with vulvar cancer, according to the procedure described elsewhere [13].

Total SCCA expression in the protein extracts of PBMC and vulvar tumours, the concentrations of SCCA (total) in culture media and 22 patients' sera were measured using the Architect SCCA kit of Abbott, and SCCA in protein extracts were expressed in nanogram per milligram of cell protein $(\mathrm{CP})$, while in the sera and culture media in nanogram per millilitre.

Immunocytochemical staining was performed using primary anti-SCCA1, anti-SCCA2 MAb (Santa Cruz, $8 \mathrm{H} 11$ and $10 \mathrm{C} 12$, respectively) and secondary anti-mouse IgG rabbit antibody Alexa 633 conjugated (Invitrogen). Nuclei were counterstained with chromomycin $\mathrm{A}_{3}$ (Sigma), and the results were imaged with confocal microscope (Axiovert $100 \mathrm{M}$, Zeiss) and LSM 510 META application.

Immunohistochemical staining was performed with antiSCCA1 (8H11), anti-SCCA2 (10C12) and anti-total SCCA (B-9) MAbs (Santa Cruz). Nuclei were counterstained with haematoxylin and eosin, and the results were imaged under a light microscope.

\section{Results}

The specific primers for total SCCA, SCCA1 and SCCA2 amplified the correct sequences, as confirmed by sequencing. As shown by the nested RT-PCR, total SCCA was expressed in mitogen-stimulated PBMC, where it persisted for all 6 days of culture, as well as in unstimulated PBMC following 1-day culture without mitogen (Fig. 1 and Table 1). SCCA was undetectable in the freshly isolated PBMC.

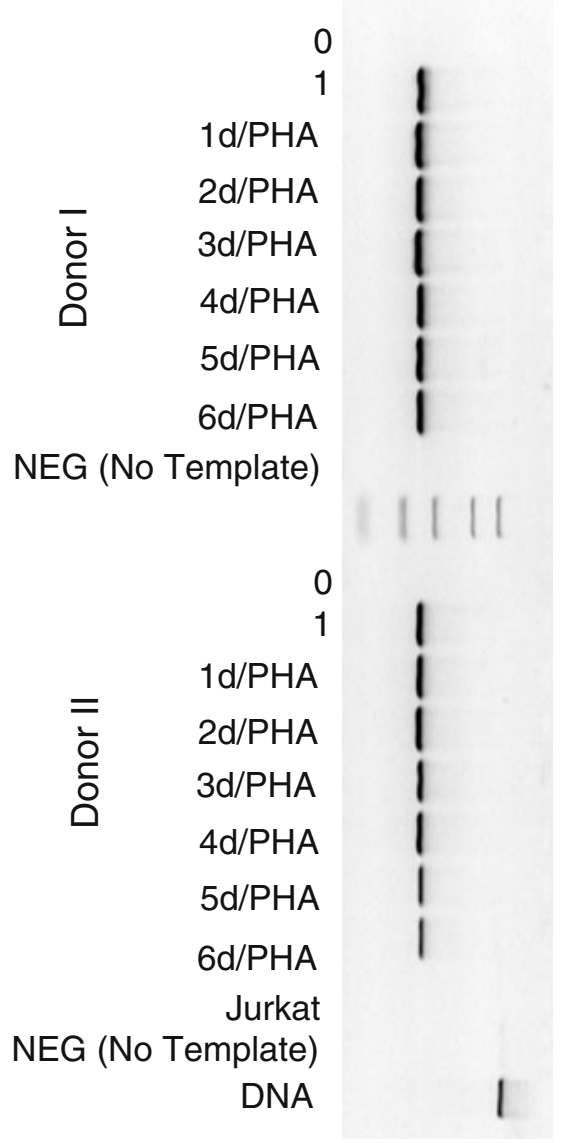

Fig. 1 Expression of total SCCA mRNA in PBMC from donor I $(0-$ before culture, 1 - unstimulated 1-day culture and PHA stimulated 1-6 day cultures)

The SCCA1 and SCCA2 genes usually co-expressed in PBMC, and this was also observed in cultures with no mitogen stimulation. In PBMC from donor I, the level of SCCA1 expression was constant up to 3 days of culture, but in the subsequent 3 days, PBMC presented no SCCAI expression, in contrast to $S C C A 2$, which was detectable throughout the whole culture period (Fig. 2 and Table 1). The level of SCCA2 expression assessed semiquantitatively in 1-day cultures was found to be lower than that of SCCA1, in both stimulated and unstimulated PBMC of donor I (Fig. 3). The total SCCA was found to be consequently expressed in PBMC of all donors throughout the culture, while the SCCA1 and SCCA2 gene expression, although found to be expressed in all donors, was identified in different time points in different donors (Table 1).

The SCCA mRNA expression in post-surgical samples from patients with VSCC was also examined. Already in one-step PCR, all 39 tumour samples were SCCA positive, while the squamous cell vulvar carcinoma cell line A431 was SCCA negative (A431 was positive in two-step PCR). The SCCA transcripts were also detected in 31 out of 36 uninvolved adjacent tissues (86\%). 
Table 1 Total SCCA, SCCA1 and SCCA2 expression, assessed by the nested RT-PCR

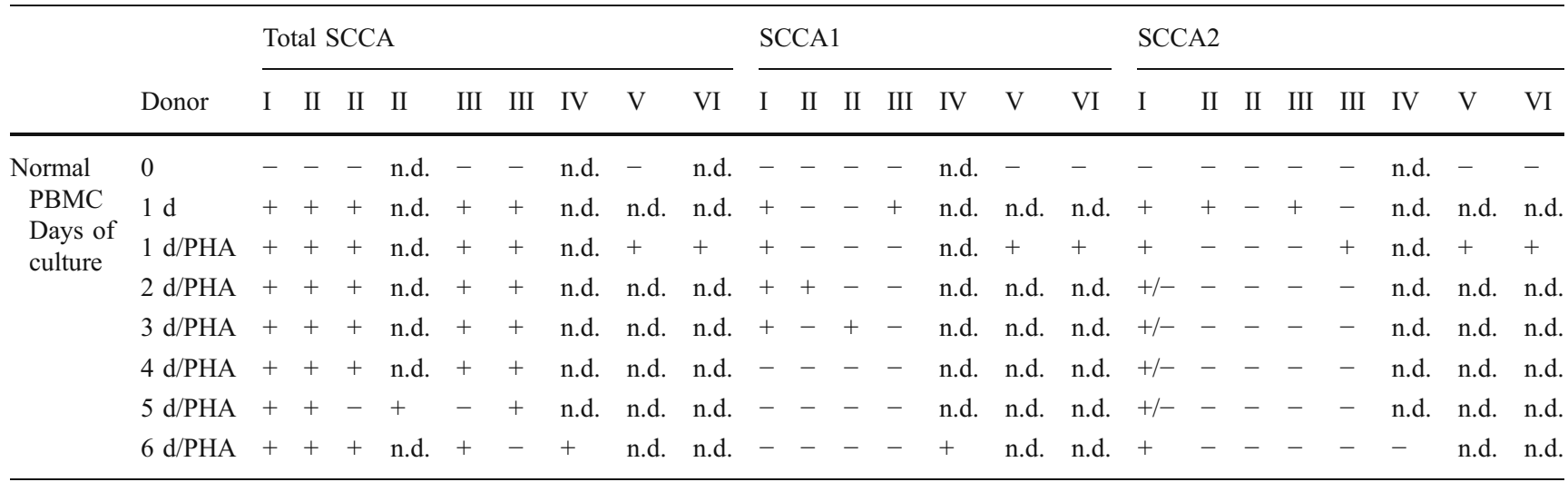

In donors II and III repeated assessments were performed

$(+)$ present, $(-)$ absent, $(+/-)$ trace expression, n.d. not determined

The SCCA concentration in the protein extract of activated PBMC was found to reach $2.7 \mathrm{ng} / \mathrm{mg}$ of CP (mean, $2.15 \mathrm{ng} / \mathrm{mg}$; Table 2). In the post-surgical samples from VSCC patients, SCCA concentrations were two to four orders of magnitude higher than those in cultured PBMC. The SCCA protein concentrations were usually higher in the tumour than in the uninvolved adjacent tissue (Table 2). Metastatic inguinal lymph nodes expressed higher levels of SCCA protein than uninvolved inguinal lymph nodes derived from the same patients. Interestingly,

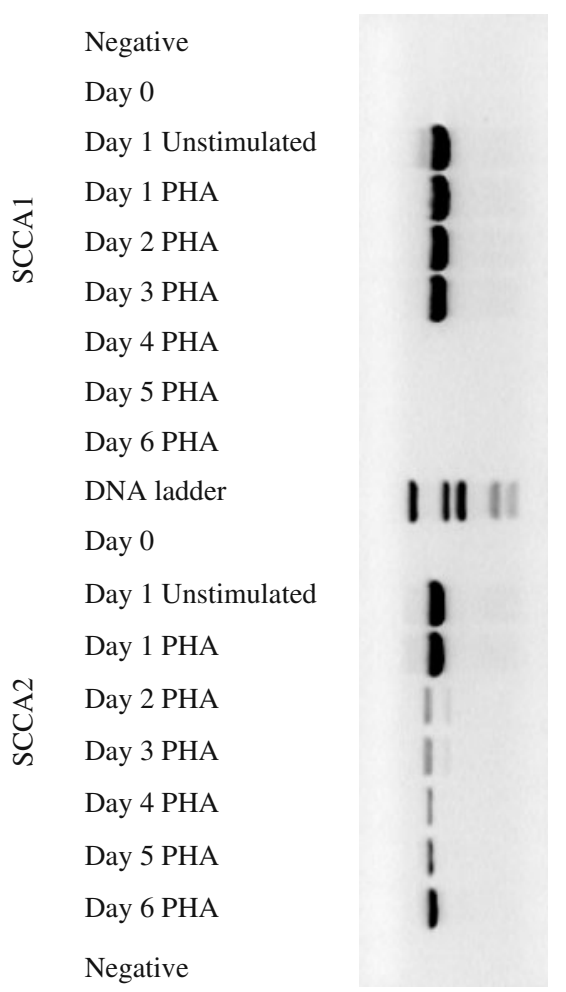

Fig. 2 SCCA1 and SCCA2 mRNA expression kinetics in stimulated PBMC from donor I in patients 12 and 30, the lymph nodes that had been classified histpathologically as uninvolved (Table 2) presented relatively high SCCA levels. This might suggest that these lymph nodes did contain micrometastases.

Immunocytochemical analysis under confocal microscope has shown traces of SCCA 1 and 2 proteins in cultured PBMC (Fig. 4). As shown in donor III, the SCCA1 and 2 expression at the protein level was detected in PBMC later after stimulation than at the mRNA level (Table 3).

The immunohistochemical analysis of tumour and inguinal lymph node from a patient with VSCC confirmed the evidently stronger SCCA staining in the metastatic lymph node than in the SCC sample (Fig. 5) and showed that not only cancer cells express SCCA protein (Table 4).

SCCA secretion was examined in cell cultures. SCCA was undetectable in the spent media from PBMC cultures, as well as from HaCat keratinocyte cell line $(<0.3 \mathrm{ng} / \mathrm{ml})$, while it was secreted in relatively high amounts by the squamous cervical cancer cell line, SKG-IIIa $(8 \mathrm{ng} / \mathrm{ml})$, known for high SCCA expression level.

The median SCCA level in the sera of 22 patients with early VSCC was $1.35 \mathrm{ng} / \mathrm{ml}$ (7.4-0.6). Only four (18\%) of

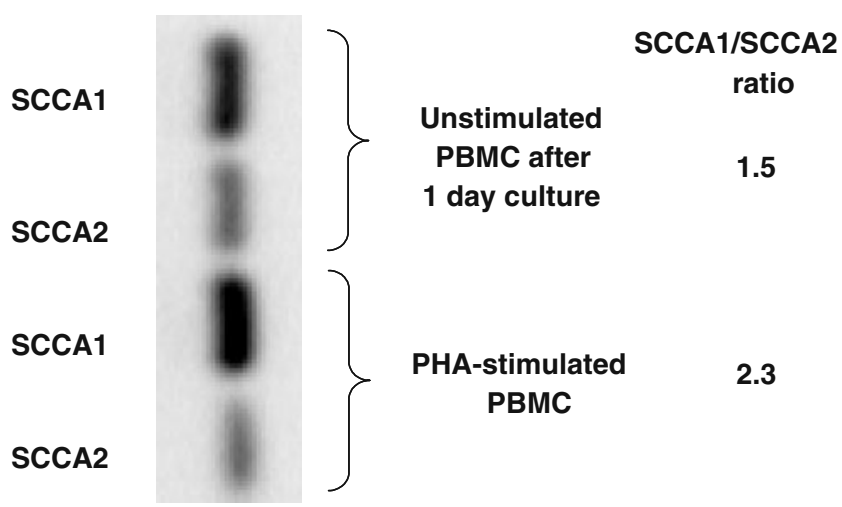

Fig. 3 Semiquantitative assessment of SCCA1/SCCA2 expression ratio in PBMC 
Table 2 SCCA concentrations (Abbott IMX SCCA assay) in the protein extracts of normal unstimulated (day 0) and stimulated (day 3 ) peripheral blood mononuclear cells (PBMC) and of tissue samples as well as in the sera from patients with VSCC

\begin{tabular}{|c|c|c|c|c|c|c|c|c|c|c|c|}
\hline \multirow[b]{2}{*}{ Healthy donor PBMC } & \multicolumn{4}{|c|}{ Donor number } & \multicolumn{7}{|c|}{ Patient number } \\
\hline & I & II & III & IV & & & & & & & \\
\hline Unstimulated & 0 & 0 & 0 & 0 & & & & & & & \\
\hline Stimulated & 2.7 & 2.7 & 1.4 & 1.8 & & & & & & & \\
\hline Samples of VSCC & & & & & 12 & 13 & 15 & 30 & 34 & 38 & 53 \\
\hline Tumour & & & & & 850 & 4890 & 19000 & 1850 & 6000 & 481 & 1600 \\
\hline Adjacent uninvolved tissue & & & & & 2920 & 290 & 2000 & 946 & 1650 & 194 & 818 \\
\hline Metastatic lymph node & & & & & $\mathrm{x}$ & $\mathrm{x}$ & 35300 & 810 & 3600 & 609 & 579 \\
\hline \multirow[t]{3}{*}{ Uninvolved lymph node } & & & & & $0.3^{\mathrm{a}}$ & $0^{\mathrm{a}}$ & 24 & 600 & 274 & 2.5 & 0 \\
\hline & & & & & 740 & 0.7 & & & & & \\
\hline & & & & & & 0 & & & & & \\
\hline Serum & & & & & n.d & 0.9 & n.d. & n.d. & 5.6 & 1.8 & 2 \\
\hline
\end{tabular}

Concentrations of SCCA in the cells and post-surgical samples are presented in nanogram per milligram of cell protein and in the sera in nanogram per millilitre of serum

$x$ no metastatic nodes found, n.d. not determined

${ }^{\mathrm{a}}$ More than one lymph node of this patient was tested

those patients had elevated SCCA levels, and three had SCCA at the cut-off level $(2 \mathrm{ng} / \mathrm{ml})$.

\section{Discussion}

We were the first to show the SCCA expression to be inducible in normal PBMC [18]. Here, we studied the total SCCA expression along with the expression of its acidic and neutral forms in more detail. We confirmed our earlier results [18] and those of Catatelpe [12], who studied different normal tissues, that SCCA mRNA is not expressed in uncultured normal PBMC. However, as we showed here, normal, activated PBMCs express SCCA1 and SCCA2 at both the mRNA and protein level. The two SCCA forms are often identified in different time points following stimulation, both in protein and mRNA studies. Interestingly, also unstimulated PBMCs following culture in the presence of FCS were found to express SCCA1 and SCCA2 trancripts. In addition, both in unstimulated (cultured for 1 day without mitogen) and in stimulated normal PBMC, the SCCA1 expression measured at the mRNA level was higher than the SCCA2 expression. Our semiquantitative assessment of the relative SCCA1 and SCCA2 expression revealed that

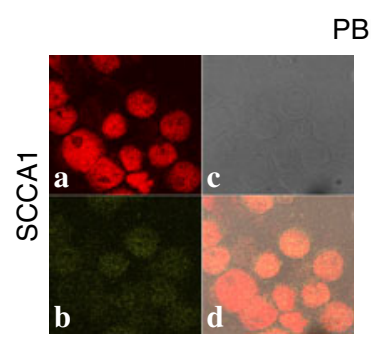

PBMC
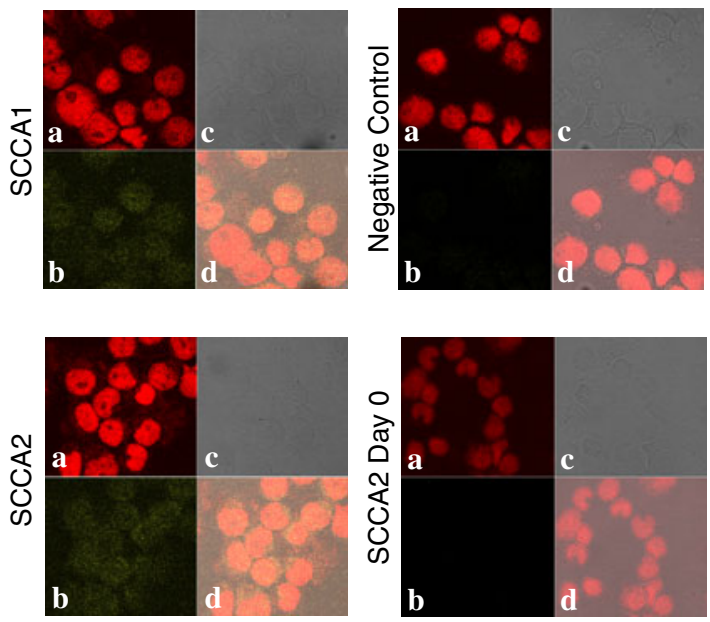

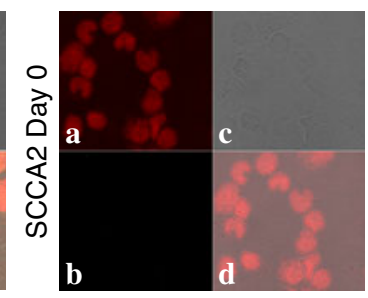

UT SCC 8
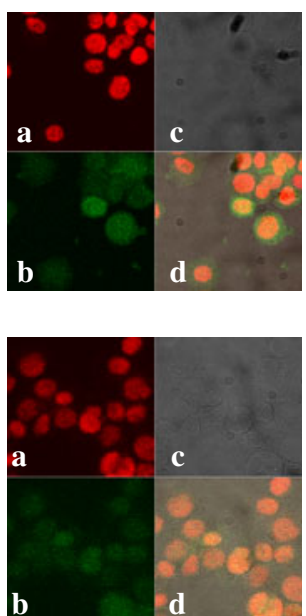

HeLa
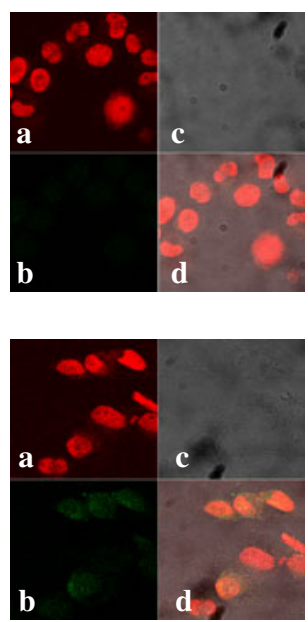

Fig. 4 SCCA1 and SCCA2 expression (green) in stimulated PBMC and UT-SCC-8 and HeLa cell lines. Confocal microscope images. a Chromomycin-stained nuclei (red), b SCCA 1 or SCCA 2 fluores- cence, $\mathbf{c}$ transmitted light images, $\mathbf{d}$ an overlay of panels $\mathbf{b}$ on $\mathbf{a}$, illustrating the cytoplasmic fluorescence

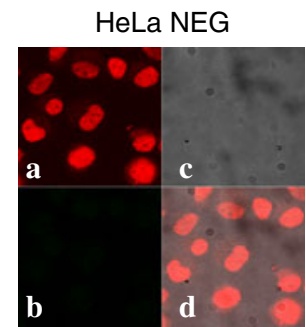

b d

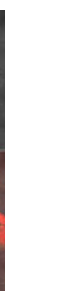


Table 3 A comparison of SCCA1 and SCCA2 expression in the peripheral blood mononuclear cells (PBMC)

mRNA expression was evaluated by nested RT-PCR, protein expression was estimated by immunocytochemistry

$(+)$ present, $(-)$ absent, $(+/-)$ trace expression
Donor III

\begin{tabular}{llllll}
\hline Normal PBMC & SCCA1 mRNA & Anti-SCCA1 & SCCA2 mRNA & Anti-SCCA2 \\
\hline 0 & - & - & - & - & - \\
$1 \mathrm{~d}$ & + & - & + & - & - \\
$1 \mathrm{~d} /$ PHA & - & - & - & + & +- \\
$2 \mathrm{~d} /$ PHA & - & +- & - & - & + \\
$3 \mathrm{~d} /$ PHA & - & - & - & - & - \\
4 d/PHA & - & + & - & - & - \\
5 d/PHA & - & $-/$ & - & - & - \\
6 d/PHA & - & - & & & - \\
\hline
\end{tabular}

the SCCA1/SCCA2 ratio increased 1.5 fold (1.54:2.38) upon PBMC stimulation. Similarly, squamous epitheliumderived cells, such as normal keratinocytes, normal nonkeratinocyte cells, cervical squamous cell carcinoma and adenocarcinoma cells, have been shown to express higher mRNA levels of SCCA1 than of SCCA2 [20]. However, in squamous cell carcinomas of head and neck, Stenman et al. [15] observed a decrease in SCCA1/SCCA2 expression ratio in malignant squamous cells; that is, the SCCA1/ SCCA2 mRNA ratios were lower in tumour tissue (median ratio $<5)$ than in normal epithelium (median ratio $>10$ ). Those ratios were also lower in primary tumours that developed into recurrent disease and in recurrent secondary tumours than in non-recurring primary tumours. Similarly, Hsu et al. [16] found that in the uterine, the SCCA1/SCCA2 mRNA ratios were progressively decreased in normal vs. dysplastic and dysplastic vs. cancer cells. In addition, the decreased SCCA1/SCCA2 ratio carried a higher risk for recurrence in early-stage uterine cervical cancer.

The published data on SCCA expression in normal human tissues are inconsistent. In the squamous cells of normal epithelia, some authors reported the SCCA immunoreactivity [21, 22], while others [23, 24] detected low or absent SCCA expression. The discrepancies might result from the different antibody specificities and tissue origins. Cataltepe et al. [12] have shown stronger SCCA1 and SCCA2 immunoreactivity in normal skin over areas of
Fig. 5 Expression of total SCCA and its forms (SCCA1 and SCCA2) in the parafin sections of VSCC and metastatic lymph node sections. Immunohistochemical staining (Santa Cruz MAb)

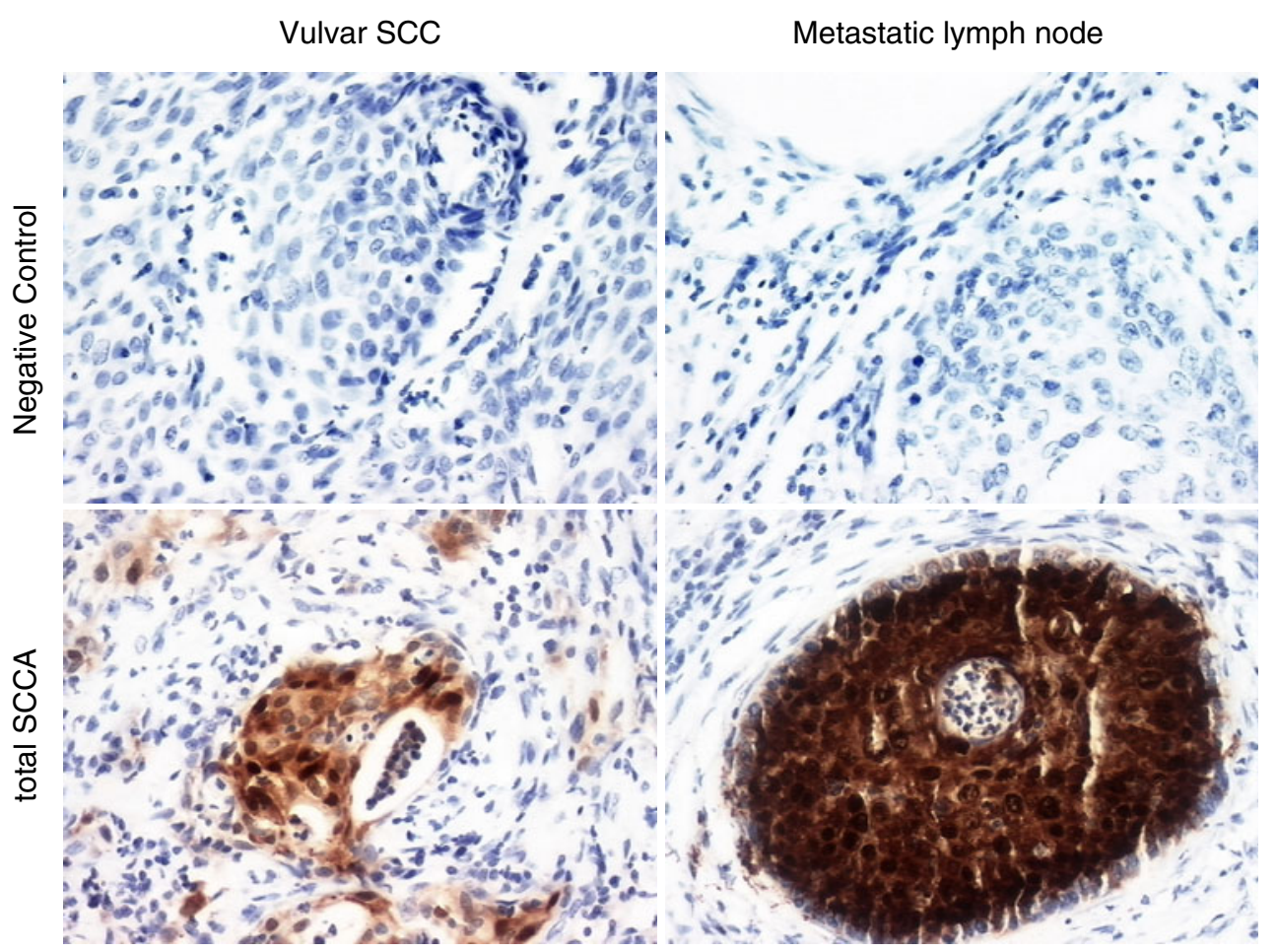


Table 4 Total SCCA, SCCA1 and SCCA2 expression according to cell types, identified by immunohistochemical examination of vulvar tumour and inguinal lymph node sections

$T$ tumour, $N$ inguinal lymph node, 0 no such cells identified, $(+)$ present, $(-)$ absent

\begin{tabular}{|c|c|c|c|c|c|c|c|c|c|c|}
\hline & \multicolumn{10}{|c|}{ Cell types } \\
\hline & \multicolumn{2}{|c|}{ Cancers } & \multicolumn{2}{|c|}{ Granulocyte } & \multicolumn{2}{|c|}{ Lymphocytes } & \multicolumn{2}{|c|}{ Plasmocytes } & \multicolumn{2}{|c|}{ Normal basal epithelial } \\
\hline & $\mathrm{T}$ & $\mathrm{N}$ & $\mathrm{T}$ & $\mathrm{N}$ & $\mathrm{T}$ & $\mathrm{N}$ & $\mathrm{T}$ & $\mathrm{N}$ & $\mathrm{T}$ & $\mathrm{N}$ \\
\hline Total SCCA & + & + & - & - & - & - & - & - & - & 0 \\
\hline SCCA1 & - & - & + & 0 & - & - & - & 0 & - & 0 \\
\hline SCCA2 & + & + & + & 0 & - & - & - & 0 & + & 0 \\
\hline
\end{tabular}

inflammation. This observation indicates that the expression of SCCA in the skin can be induced by local inflammation. Indeed, SCCA has been recognised as a marker of inflammatory skin diseases [23, 25, 26].

Interestingly, in the squamous cell carcinomas of the tongue, Yasumatsu et al. [27] observed SCCA1 immunostaining in the T-lymphocytes peripheral to cancer cells and found this staining, but not SCCA1 expression in cancer cells, to significantly correlate with elevated serum SCCA concentrations. We showed here SCCA immunoreactivity in vulvar tumour-associated granulocytes. Thus, the source of serum SCCA may not necessarily be cancer cells but other tumour-associated cells, such as infiltrating immune cells. Elevated serum SCCA associated with several nonneoplastic dermatological diseases [23, 25] as well as with renal failure or lung diseases [28] support the possible role of different non-neoplastic cells in SCCA release. Furthermore, Van der Sijde et al. [29], who found serum SCCA assessment a useful tool for the early detection of recurrent VSCC, also observed $25 \%$ of false-positive (i.e. in patients with no cancer) SCCA results in patients with benign skin disorders. In addition, elevated concentrations of serum SCCA and/or C-reactive protein were reported to be independent unfavourable prognostic factors in head and neck squamous cell carcinomas [30], which links SCCA with systemic symptoms of cancer-related inflammation. Taken together, these data suggest that SCCA is not only a marker of squamous cell carcinomas but also of inflammatory conditions, and although high sensitivity of serum SCCA determination in cancer patients is generally appreciated, at the same time its limited specificity is evident.

Here, we also examined SCCA expression in VSCC patients. The levels of SCCA protein and mRNA induced in normal PBMC were several orders of magnitude lower than those found in the vulvar tumour cells and in the cells of tumour-adjacent tissues and lymph nodes. However, high levels of SCCA in the local tumour microenvironment and regional metastatic sites in patients with VSCC seem not to imply elevated serum SCCA levels, as few of those patients had serum SCCA concentrations above the normal level. In patients with early stage VSCC, consistently with our data, other researchers have shown mean serum SCCA level to be below the cut-off value [4]. Serum SCCA levels were found not to be associated with lymph node involvement [4, 29, 31]. In advanced VSCC (FIGO stage IV), less than $40 \%$ of patients present elevated serum SCCA concentrations [4, 29].

The origin of serum SCCA in patients with SCC remains an open question. Uemura et al. [13] have shown the lack of SCCA secretion by the SCC cells, but other studies contradicted this result $[32,33]$. Our analysis of SCCA concentrations in the spent media of stimulated PBMC, and of the squamous epithelia-derived cells of the HaCat and SKG-IIIa cervical cancer cell lines, showed SCCA in the culture media of SKG-IIIa cells but not of PBMC or HaCat cells. Thus, SCCA identified in body fluids may either be secreted by the cells and/or may derive from dying cells. In addition, SCCA expression and secretion is inducible by the Th2 cytokines, IL-13 and IL-4 in normal squamous epithelial cells [26, 33-35]. The Th1 to Th2 shift is regarded as typical of cancer; still, only SCC patients present elevated serum SCCA concentrations. Therefore, we hypothesise that an increase in the serum SCCA levels in patients with squamous cell carcinomas may be associated with SCCA induction by cytokines in normal squamous epithelial cells present in SCC microenvironment.

In summary, normal, activated PBMCs express SCCA1 and SCCA2 at both the mRNA and protein level, but the two forms of SCCA are not necessarily expressed concurrently. We could not demonstrate that cancer-related inflammatory cells are potentially a significant source of circulating SCCA in VSCC. In vulvar SCC patients, not only tumours but also metastatic lymph nodes are potential sources of SCCA protein. We think that the raised levels of serum SCCA observed by some researchers in the advanced stages of vulvar carcinoma [36] may result from secondary changes related to the disease progression, such as alterations induced by metastatic cancer cells in their microenvironment and/or an increase in proinflammatory cytokines. These changes create a feedback between local and systemic inflammation, a phenomenon that we have discussed elsewhere [37]. The exact mechanisms of releasing of SCCA into the body fluids remain to be resolved. 
Acknowledgements The authors would like to thank Dr Małgorzata Jarmuż, Institute of Human Genetics, Polish Academy of Sciences, Poznan, Poland and Prof. Reidar Grenman, University of Turku, Finland, for providing the UT-SCC-8 cell line, and Prof. Krzysztof Włodarski, Warsaw Medical University, Warsaw, Poland, for providing the HeLa cell line.

Open Access This article is distributed under the terms of the Creative Commons Attribution Noncommercial License which permits any noncommercial use, distribution, and reproduction in any medium, provided the original author(s) and source are credited.

\section{References}

1. Kato H, Torigoe T. Radioimmunoassay for tumor antigen of human cervical squamous cell carcinoma. Cancer. 1977;40:1621-8.

2. Suminami Y, Kishi F, Sekiguchi K, Kato H. Squamous cell carcinoma antigen is a new member of the serine protease inhibitors. Biochem Biophys Res Commun. 1991;181:51-8.

3. Torre GC. SCC antigen in malignant and nonmalignant squamous lesions. Tumour Biol. 1998;19:517-26.

4. Hefler LA, Sliutz G, Leodolter S, Speiser P, Joura E, Reinthaller A, et al. Squamous cell carcinoma antigen serum levels as prognostic parameter in patients with early stage vulvar cancer. Gynecol Oncol. 2005;97:904-7.

5. Lara PC, Cuyas JM. The role of squamous cell carcinoma antigen in the management of laryngeal and hypopharyngeal cancer. Cancer. 1995;76:758-64.

6. Molina R, Torres MD, Moragas M, Perez-Villa J, Filella X, Jo J, et al. Prognostic significance of SCC antigen in the serum of patients with head and neck cancer. Tumour Biol. 1996;17:81-9.

7. Sanchez De Cos J, Masa F, de la Cruz JL, Disdier C, Vergara C. Squamous cell carcinoma antigen ( $\mathrm{SCC} \mathrm{Ag)} \mathrm{in} \mathrm{the} \mathrm{diagnosis} \mathrm{and}$ prognosis of lung cancer. Chest. 1994;105:773-6.

8. Strauss HG, Laban C, Lautenschlager C, Buchmann J, Schneider I, Koelbl H. SCC antigen in the serum as an independent prognostic factor in operable squamous cell carcinoma of the cervix. Eur J Cancer. 2002;38:1987-91.

9. Gadducci A, Tana R, Cosio S, Genazzani AR. The serum assay of tumour markers in the prognostic evaluation, treatment monitoring and follow-up of patients with cervical cancer: a review of the literature. Crit Rev Oncol Hematol. 2008;66:10-20.

10. Schick C, Kamachi Y, Bartuski AJ, Cataltepe S, Schechter NM, Pemberton PA, et al. Squamous cell carcinoma antigen 2 is a novel serpin that inhibits the chymotrypsin-like proteinases cathepsin $\mathrm{G}$ and mast cell chymase. J Biol Chem. 1997;272:1849-55.

11. Schick C, Pemberton PA, Shi GP, Kamachi Y, Cataltepe S, Bartuski $\mathrm{AJ}$, et al. Cross-class inhibition of the cysteine proteinases cathepsins $\mathrm{K}, \mathrm{L}$, and $\mathrm{S}$ by the serpin squamous cell carcinoma antigen 1: a kinetic analysis. Biochemistry. 1998;37:5258-66.

12. Cataltepe S, Gornstein ER, Schick C, Kamachi Y, Chatson K, Fries J, et al. Co-expression of the squamous cell carcinoma antigens 1 and 2 in normal adult human tissues and squamous cell carcinomas. J Histochem Cytochem. 2000;48:113-22.

13. Uemura Y, Pak SC, Luke C, Cataltepe S, Tsu C, Schick C, et al. Circulating serpin tumor markers SCCA1 and SCCA2 are not actively secreted but reside in the cytosol of squamous carcinoma cells. Int J Cancer. 2000;89:368-77.

14. Roijer E, de Bruijn HW, Dahlen U, ten Hoor K, Lundin M, Nilsson $\mathrm{K}$, et al. Squamous cell carcinoma antigen isoforms in serum from cervical cancer patients. Tumour Biol. 2006;27:142-52.

15. Stenman J, Hedstrom J, Grenman R, Leivo I, Finne P, Palotie A, et al. Relative levels of SCCA2 and SCCA1 mRNA in primary tumors predicts recurrent disease in squamous cell cancer of the head and neck. Int J Cancer. 2001;95:39-43.

16. Hsu KF, Huang SC, Shiau AL, Cheng YM, Shen MR, Chen YF, et al. Increased expression level of squamous cell carcinoma antigen 2 and 1 ratio is associated with poor prognosis in earlystage uterine cervical cancer. Int J Gynecol Cancer. 2007;17: 174-81.

17. Stenman J, Lintula S, Hotakainen K, Vartiainen J, Lehvaslaiho H, Stenman UH. Detection of squamous-cell carcinoma antigenexpressing tumour cells in blood by reverse transcriptasepolymerase chain reaction in cancer of the uterine cervix. Int $\mathrm{J}$ Cancer. 1997;74:75-80.

18. Kowalewska M, Chechlinska M, Markowicz S, Kober P, Nowak R. The relevance of RT-PCR detection of disseminated tumour cells is hampered by the expression of markers regarded as tumour-specific in activated lymphocytes. Eur J Cancer. 2006;42:2671-4

19. Boukamp P, Petrussevska RT, Breitkreutz D, Hornung J, Markham A, Fusenig NE. Normal keratinization in a spontaneously immortalized aneuploid human keratinocyte cell line. J Cell Biol. 1988;106:761-71.

20. Hamada K, Zhang T, Desaki J, Nakashiro K, Ito H, Tani K, et al. Carrier cell-mediated cell lysis of squamous cell carcinoma by squamous cell carcinoma antigen 1 promoter-driven oncolytic adenovirus. Nature Precedings 2009. http://precedings.nature. com/documents/3213/version/1.

21. Mino-Miyagawa N, Kimura Y, Hamamoto K. Tumor-antigen 4. Its immunohistochemical distribution and tissue and serum concentrations in squamous cell carcinoma of the lung and esophagus. Cancer. 1990;66:1505-12.

22. Horiuchi Y, Tsukahara T, Otoyama K. Immunohistochemical study of elevated expression of squamous cell carcinoma (SCC)related antigens in erythrodermic epidermis. J Dermatol. 1994;21:67-72.

23. Duk JM. van Voorst Vader PC, ten Hoor KA, Hollema H, Doeglas HM, de Bruijn HW. Elevated levels of squamous cell carcinoma antigen in patients with a benign disease of the skin. Cancer. 1989;64:1652-6.

24. Morioka H. Tumor-antigen (TA-4) of squamous cell carcinomaits tissue distribution and its relationship to serum TA-4 concentrations. Asia Oceania J Obstet Gynecol. 1980;6:91-7.

25. Campbell B, De'Ambrosis B. Squamous cell carcinoma antigen in patients with cutaneous disorders. J Am Acad Dermatol. 1990;22:639-42.

26. Mitsuishi K, Nakamura T, Sakata Y, Yuyama N, Arima K, Sugita $\mathrm{Y}$, et al. The squamous cell carcinoma antigens as relevant biomarkers of atopic dermatitis. Clin Exp Allergy. 2005;35:1327-33.

27. Yasumatsu R, Nakashima T, Azuma K, Hirakawa N, Kuratomi Y, Tomita K, et al. SCCA1 expression in T-lymphocytes peripheral to cancer cells is associated with the elevation of serum SCC antigen in squamous cell carcinoma of the tongue. Cancer Lett. 2001;167:205-13.

28. Molina R, Filella X, Torres MD, Ballesta AM, Mengual P, Cases $\mathrm{A}$, et al. SCC antigen measured in malignant and nonmalignant diseases. Clin Chem. 1990;36:251-4.

29. van der Sijde R, de Bruijn HW, Krans M, Bouma J, Aalders JG. Significance of serum SCC antigen as a tumor marker in patients with squamous cell carcinoma of the vulva. Gynecol Oncol. 1989;35:227-32.

30. Kulpa J, Stasik Z, Skolyszewski J, Wojcik E, Rychlik U, Pudelek K. Predictive value of SCC-Ag, CYFRA 21-1 and selected acute phase proteins in radiotherapy of pharyngeal and laryngeal cancer. A preliminary report. Neoplasma. 2004;51:103-9.

31. Nather A, Sami A, Hefler L, Leodolter S, Joura EA. Serum levels of squamous cell carcinoma antigen as a predictor of inguinofe- 
moral lymph node metastasis in patients with vulvar cancer. $\mathrm{J}$ Reprod Med. 2002;47:718-20.

32. Hamada K, Hanakawa Y, Hashimoto K, Iwamoto M, Kihana T, Hirose $\mathrm{S}$, et al. Gene expression of human squamous cell carcinoma antigens 1 and 2 in human cell lines. Oncol Rep. 2001;8:347-54.

33. Kanaji S, Tanaka Y, Sakata Y, Takeshita K, Arima K, Ohta S, et al. Squamous cell carcinoma antigen 1 is an inhibitor of parasitederived cysteine proteases. FEBS Lett. 2007;581:4260-4.

34. Ray R, Choi M, Zhang Z, Silverman GA, Askew D, Mukherjee AB. Uteroglobin suppresses SCCA gene expression associated with allergic asthma. J Biol Chem. 2005;280:9761-4.
35. Yuyama N, Davies DE, Akaiwa M, Matsui K, Hamasaki Y, Suminami Y, et al. Analysis of novel disease-related genes in bronchial asthma. Cytokine. 2002;19:287-96.

36. Hefler L, Frischmuth K, Heinze G, Sliutz G, Leodolter S, Reinthaller A, et al. Serum concentrations of squamous-cell carcinoma antigen and tissue polypeptide antigen in the follow-up of patients with vulvar cancer. Int J Cancer. 1999;83:167-70.

37. Chechlinska M, Kowalewska M, Nowak R. Systemic inflammation as a confounding factor in cancer biomarker discovery and validation. Nat Rev Cancer. 2010;10:2-3. 